Submitted to The ApJL

Preprint typeset using $\mathrm{LAT}_{\mathrm{E}} \mathrm{X}$ style emulateapj v. 5/2/11

\title{
ATMOSPHERIC CIRCULATION OF BROWN DWARFS: JETS, VORTICES, AND TIME VARIABILITY
}

\author{
Xi Zhang AND AdAm P. ShOWMAN \\ Department of Planetary Sciences and Lunar and Planetary Laboratory, University of Arizona, AZ 85721 \\ submitted to the ApJL
}

\begin{abstract}
A variety of observational evidence demonstrates that brown dwarfs exhibit active atmospheric circulations. In this study we use a shallow-water model to investigate the global atmospheric dynamics in the stratified layer overlying the convective zone on these rapidly rotating objects. We show that the existence and properties of the atmospheric circulation crucially depend on key parameters including the energy injection rate and radiative timescale. Under conditions of strong internal heat flux and weak radiative dissipation, a banded flow pattern comprising east-west jet streams spontaneously emerges from the interaction of atmospheric turbulence with the planetary rotation. In contrast, when the internal heat flux is weak and/or radiative dissipation is strong, turbulence injected into the atmosphere damps before it can self-organize into jets, leading to a flow dominated by transient eddies and isotropic turbulence instead. The simulation results are not very sensitive to the form of the forcing. Based on the location of the transition between jet-dominated and eddy-dominated regimes, we suggest that many brown dwarfs may exhibit atmospheric circulations dominated by eddies and turbulence (rather than jets) due to the strong radiative damping on these worlds, but a jet structure is also possible under some realistic conditions. Our simulated light curves capture important features from observed infrared lightcurves of brown dwarfs, including amplitude variations of a few percent and shapes that fluctuate between single-peak and multi-peak structures. More broadly, our work shows that the shallow-water system provides a useful tool to illuminate fundamental aspects of the dynamics on these worlds.

Subject headings: brown dwarfs - stars: low-mass - stars: atmospheres - turbulence - methods: numerical
\end{abstract}

\section{INTRODUCTION}

Brown dwarfs are characterized by a vigorously convective interior overlain by a stratified weather layer (Burrows et al. 2006). Increasing observational evidence, including chemical disequilibrium (e.g., Saumon et al. 2006; Leggett et al. 2007), condensates and clouds (e.g., Tsuji 2002; Knapp et al. 2004), infrared flux variability (e.g., Artigau et al. 2009; Radigan et al. 2012; Apai et al. 2013), and surface patchiness (Crossfield et al. 2014) imply the existence of strong vertical mixing and large-scale atmospheric motion on brown dwarfs. To understand the atmospheric convection and circulation, several models have been put forward. A local-box simulation by Freytag et al. (2010) showed that convection could trigger gravity waves in the stratified layer, causing vertical mixing and helping maintain thin dust clouds. Showman \& Kaspi (2013) presented the first three-dimensional (3D) general circulation model of brown dwarfs and demonstrated that the atmosphere and interior dynamics are rotationally dominated. However, many aspects of the expected atmospheric dynamics remain uncertain. Especially, there is a lack of global simulations investigating the stratified layer on brown dwarfs.

The surface patchiness suggested in the observations raises major questions about the nature of the atmospheric circulation on brown dwarfs. In particular, are the flows on brown dwarfs zonally banded, consisting of numerous east-west jet streams like those on Jupiter and Saturn? Or do they consist primarily of turbulence and eddies with no preferred directionality? The issue has

Contact email: xiz@lpl.arizona.edu major implications for interpretations of variability.

To answer these questions, we introduce an idealized global shallow-water model to study the atmospheric circulation on brown dwarfs. The shallow water model has been extensively used in the atmospheric studies on Earth (e.g., Sadourny 1974), giant planets (e.g., Cho and Polvani 1996; Showman 2007) and exoplanets (Showman \& Polvani 2011). Ours are the first global numerical simulations of the dynamics in the stratified atmospheres of brown dwarfs.

\section{SHALLOW WATER MODEL AND THE BROWN DWARF REGIME}

We adopt a two-layer model, with constant densities in each layer. The upper layer, of lower density, represents the flow in the stratified atmosphere (pressure less than several bars), and the lower layer, of greater density, represents the convective interior. The interface between the layers represents an isentrope near the radiative-convective boundary $(\mathrm{RCB})$. In the limit where the lower layer is quiescent and infinitely deep, this system reduces to the one-layer shallow-water equations for the flow in the upper layer (e.g., Vallis 2006)

$$
\begin{array}{r}
\frac{D \mathbf{u}}{D t}+\nabla(g h)+f \mathbf{k} \times \mathbf{u}=\mathbf{R} \\
\frac{D(g h)}{D t}+g h \nabla \cdot \mathbf{u}=g \frac{h_{\mathrm{eq}}-h}{\tau_{\text {rad }}}+S
\end{array}
$$

where $\mathbf{u}$ is the horizontal velocity vector, $f=2 \Omega \sin \phi$ is Coriolis parameter, $\Omega$ is the rotation rate, $\phi$ is latitude, $\mathbf{k}$ is the upward unit vector, $g h$ is the geopotential, where $g$ 
is gravity and $h$ is the layer thickness, and $\mathbf{R}$ is an advection term required to account for momentum transport between the layers (Showman \& Polvani 2011).

The forcing term $S$ represents the exchanges of mass, such as convective overshoot, across the RCB (see Section 2.2 below). Radiative heating and cooling are represented by a Newtonian cooling scheme, which relaxes the layer thickness to a specified, constant radiativeequilibrium thickness $h_{\mathrm{eq}}$ on a timescale $\tau_{\mathrm{rad}}$.

Our brown dwarf shallow-water model is modified from the NCAR Spectral Transform Shallow Water Model (STSWM, Hack \& Jakob 1992), which solves the shallowwater equations using the spectral transform method in spherical geometry on the entire globe. The spatial resolution is $0.7^{\circ}$ in longitude and latitude. $\mathrm{A} \nabla^{6}$ hyperviscosity is used to maintain numerical stability. More details are available in Hack \& Jakob (1992). The initial condition is a flat layer of thickness $h_{\mathrm{eq}}$ with no winds. Next, we will introduce several important parameters identifying the brown dwarf regime.

\subsection{Deformation Radius and Layer Thickness}

The Rossby deformation radius $\left(L_{R}\right)$ measures the horizontal length scale within which the atmospheric flow is more significantly influenced by buoyancy rather than rotation. In a three-dimensional stratified atmosphere, $L_{R}=N H_{p} / f$, where $H_{p}=R_{g} T / \mu g$ is the pressure scale height, $R_{g}$ is the gas constant, $T$ is temperature, and $\mu$ is the mean molecular mass. $N=\left[g / T\left(g / c_{p}+d T / d z\right)\right]^{1 / 2}=$ $g\left[\gamma /\left(c_{p} T\right)\right]^{1 / 2}$ is the Brunt-Väisälä frequency of the atmosphere, where $\gamma=\left[1+\left(c_{p} / g\right) d T / d z\right]$ is a measure of the subadiabaticity of the stratified atmosphere; $\gamma=1$ for an isothermal atmosphere and $\gamma=0$ for a fully adiabatic profile. $c_{p}$ is the specific heat of the atmosphere, and $z$ is altitude. The observed rotation rates, surface gravities and temperatures for $\mathrm{L}$ and $\mathrm{T}$ dwarfs range from $10^{-4}$ to $10^{-3} \mathrm{~s}^{-1}$ (Reiners \& Basri 2008), 500-3000 m $\mathrm{s}^{-2}$ and $750-2200 \mathrm{~K}$ (Burrows et al. 2006), respectively. Given the temperature profile of a typical brown dwarf with temperature of $1000 \mathrm{~K}$ and surface gravity of 500 $\mathrm{m} \mathrm{s}^{-2}$, we estimate $\gamma \sim 0.1$ above the RCB. Provided the rotation rate $\Omega \sim 10^{-3} \mathrm{~s}^{-1}$, the deformation radius is $L_{R} \sim 1000 \mathrm{~km}$, similar to the Jupiter value (Vasavada \& Showman 2005).

We specify the reference geopotential, $g h_{\mathrm{eq}}$, so that the model's deformation radius matches the expected value. In the shallow-water system, $L_{R}=\sqrt{g h_{\mathrm{eq}}} / f$, implying that:

$$
g h_{e q}=\left(f L_{R}\right)^{2}=\gamma \kappa^{2} c_{p} T
$$

where $\kappa=R / \mu c_{p}$ is $\sim 2 / 7$ for a hydrogen-dominated atmosphere. Inserting brown-dwarf parameters implies that the appropriate value of $g h_{\mathrm{eq}}$ is $10^{5}-10^{6} \mathrm{~m}^{2} \mathrm{~s}^{-2}$.

\subsection{Forcing Parameters}

The mass source term $S$ represents all possible mechanisms by which convection perturbs the stratified layer, including perturbations of the RCB itself by the convection, convective overshoot and mixing across the RCB, and latent heat release due to condensation of silicates and iron. Since there is no single estimate of $S$, we specify its functional form parametrically in space and time. The mean value of the spatial pattern is zero and the standard deviation is $s_{m}$. Analogy with the isentropiccoordinate primitive equations (Vallis, 2006) implies that forcing amplitude $s_{m}$ relates to a three-dimensional atmosphere via

$$
s_{m} \approx g h \frac{q}{\Delta \theta}
$$

where $\Delta \theta$ is the difference in potential temperature across the weather layer $\left(\sim 10^{2} \mathrm{~K}\right.$ for a layer one scale height thick given the expected stratifications) and $q$ is the net heating rate at the RCB. Given the velocities expected by mixing length theory (Showman \& Kaspi 2013), convective overshoot should produce deviations from the radiative-equilibrium temperature just above the RCB of $\sim 1-10 \mathrm{~K}$, which for radiative time constants of $10^{5} \mathrm{~s}$ implies local, spatially varying heating rates induced by convection at the RCB of $10^{-5}-10^{-4} \mathrm{~K} \mathrm{~s}^{-1}$. Given $g h \sim 10^{6} \mathrm{~m}^{2} \mathrm{~s}^{-2}$, we expect $s_{m} \sim 0.1-1 \mathrm{~m}^{2} \mathrm{~s}^{-3}$.

We explore two schemes for the convective forcing. In the first, we assume that convection perturbs the stratified layer everywhere simultaneously and continuously in time with a small characteristic horizontal wavelength. The forcing pattern is specified by a superposition of spherical harmonics within the range of total spatial wavenumbers $n=n_{f}-\Delta n$ to $n_{f}+\Delta n$ and zonal wavenumbers between $-n$ and $n$, where $n_{f}$ is the central wavenumber of the forcing. In this study, we adopt $n_{f}=40$ and $\Delta n=2$, based on the spatial pattern of temperature maps from 3D simulations (Showman \& Kaspi 2013). At each time step, the amplitude of each spherical harmonic is randomly varied between 0 and $s_{m}$ and the orientation of each spherical harmonic is randomly varied (with a pole position of the coordinate system in which the spherical harmonic is evaluated that is random in longitude and latitude). These are summed into a forcing function $F(\lambda, \phi)$ at longitude $(\lambda)$ and latitude $(\phi)$. The standard deviation of $F$ is then normalized to $s_{m}$ at each time step. The forcing pattern, $S(\lambda, \phi, t)$, is marched forward in time as a Markov process:

$$
S(\lambda, \phi, t+\delta t)=\left(1-\alpha^{2}\right)^{1 / 2} S(\lambda, \phi, t)+\alpha F(\lambda, \phi)
$$

with a de-correlation factor $\alpha=\delta t / \tau_{s}$, where $\delta t$ is the timestep, $\tau_{s}$ is the forcing timescale.

In the second scheme, we postulate that the convection occurs episodically over a small fraction of the horizontal area, as is typical of moist convection on Earth and Jupiter. In this case, we randomly introduce isolated mass pulses that are circular Gaussians on the sphere with a radius $r_{s}$ and forcing timescale $\tau_{s}$. The forcing pattern at time $t$ associated with a given storm, $s_{\text {storm }}(\mathbf{r}, t)$, is:

$$
s_{\text {storm }}(\mathbf{r}, t)=s_{m} \exp \left[-\frac{\left|\mathbf{r}-\mathbf{r}_{\mathbf{0}}\right|^{2}}{r_{s}^{2}}-\frac{\left(t-t_{0}\right)^{2}}{\tau_{s}^{2}}\right]
$$

where $s_{m}$ is the maximum forcing amplitude occurring at time $t_{0},\left|\mathbf{r}-\mathbf{r}_{\mathbf{0}}\right|$ is the distance from the convection center $\mathbf{r}_{\mathbf{0}}$ to any given point $\mathbf{r}$ on the sphere. The contributions of different storms, each occurring at different locations $\mathbf{r}_{\mathbf{0}}$ and times $t_{0}$, are summed to obtain the total forcing, $S(\mathbf{r}, \mathbf{t})=\sum s_{\text {storm }}(\mathbf{r}, t)$. The mass pulses are introduced randomly over the globe and in time (see Showman 2007 for details).

The forcing timescale is uncertain. Under typical brown dwarf conditions, convective transport times 
across a scale height are $\sim 10^{3} \mathrm{~s}$, but the convection (and the mixing and wave breaking resulting from it above the RCB) may organize on longer timescales, much as cumulus convection in Earth's tropics organizes across a wide range of spatial and temporal scales (e.g., Mapes et al. 2006; Kiladis et al. 2009). We consider $\tau_{s} \sim 10^{3}-10^{5} \mathrm{~s}$.

Energetically, the forcing drives the circulation by generating available potential energy ${ }^{1}(\mathrm{APE})$, which is converted to kinetic energy associated with large-scale winds. The production rate of APE due to a single gaussian mass pulse averaged over space and cell lifetime is $\sim \pi^{2} \tau_{s} r_{s}^{2} s_{m}^{2} / 4$ (Showman 2007). For a globally convective system like a brown dwarf atmosphere, we estimate $\sim A \tau_{s} s_{m}^{2}$, where $A$ is the global surface area. We define the energy injection rate $(\epsilon)$ as the ratio of the $\mathrm{APE}$ production rate to the total potential energy of the equilibrium state, $\sim A\left(g h_{e q}\right)^{2} / 2$. With our estimated forcing parameters, the energy injection rate on a typical Jupiter-size brown dwarf is $\sim 10^{-8}-10^{-5} \mathrm{~s}^{-1}$. By comparison, $\epsilon$ of Jovian system is $\sim 10^{-11} \mathrm{~s}^{-1}$.

\subsection{Radiative Relaxation}

The radiative relaxation timescale $\left(\tau_{\text {rad }}\right)$ is estimated as:

$$
\tau_{\text {rad }} \sim \frac{p}{g} \frac{c_{p}}{4 \sigma T^{3}}
$$

where $p$ is the pressure of the typical IR photosphere level (optical depth $\sim 1$ ). For a typical brown dwarf of $\sim 1000$ $\mathrm{K}$, the radiative timescale is $\sim 10^{5} \mathrm{~s}$. Since the effective temperature of brown dwarfs ranges from several hundred (e.g., Y type) to several thousand kelvins(e.g., L0 type), the radiative timescale can vary by several orders of magnitude $\left(\sim 10^{4}-10^{7} \mathrm{~s}\right)$. By comparison, the radiative relaxation timescale for Jupiter is $\sim 10^{9} \mathrm{~s}$. Radiative cooling might be also important for the cloud formation on brown dwarfs (Helling et al. 2001).

\section{JETS VERSUS ISOTROPIC TURBULENCE ON BROWN DWARFS}

We find two major regimes of behavior depending on the strengths of the forcing and dissipation. For strong energy injection and weak dissipation, large-scale eastwest (zonal) jet streams ${ }^{2}$ emerge, particularly at the equator, where they dominate the flow. In contrast, for weak energy injection and strong dissipation, jets are absent, and eddies and turbulence dominate the dynamics. Here we show two idealized simulations with $n_{f}=40$, with a rotation period of 1.6 hours and an equilibrium geopotential of $5 \times 10^{5} \mathrm{~m}^{2} \mathrm{~s}^{-2}$ (Fig. 1). Case A, corresponding to a cold brown dwarf atmosphere $\left(\tau_{\text {rad }} \sim 10^{7} \mathrm{~s}\right)$ with $\tau_{s}=10^{5} \mathrm{~s}$ and $s_{m}=0.1 \mathrm{~m}^{2} \mathrm{~s}^{-3}$, exhibits a strong equatorial westward jet with peak wind velocity of $50 \mathrm{~m} \mathrm{~s}^{-1}$ and long-lived vortices in the middle and high latitudes. Case B corresponds to a hotter brown dwarf atmosphere $\left(\tau_{\text {rad }} \sim 10^{5} \mathrm{~s}\right)$ with $\tau_{s}=10^{3}$ $\mathrm{s}$ and $s_{m}=0.1 \mathrm{~m}^{2} \mathrm{~s}^{-3}$, implying that the energy injection rate is 100 times weaker and the dissipation is 100 times stronger than in case A. As a result, transient eddies and isotropic turbulence, rather than jets, dominate

\footnotetext{
${ }^{1}$ APE is the difference in potential energy between the stratified atmosphere and a reference state (Vallis, 2006).

2 i.e., zonal mean zonal wind that exceeds the standard deviation of zonal wind in longitude
}

the entire globe. The geopotential variation in case $\mathrm{A}$ is almost a factor of 10 larger than that in case B. Over the ranges of rotation rate $\left(10^{-3}-10^{-4} \mathrm{~s}^{-1}\right)$ and spectral wavenumber (20-70) we explored, the system shows similar behavior.

To systematically investigate the dichotomy of jet- versus turbulence-dominated, we performed several hundred simulations over a wide range of forcing and dissipation parameters. Atmospheres with an equilibrium geopotential of $10^{5} \mathrm{~m}^{2} \mathrm{~s}^{-2}$ were forced by either local mass pulses with randomly-chosen size, strength, and lifetime, or global spectral forcing with spherical harmonics $\left(n_{f}=40\right)$ and random forcing amplitude. In the $\epsilon-\tau$ coordinate plane (Fig. 2), all cases fall into two distinct regimes: with or without jets. The two regimes are separated by a boundary line with slope of $\sim 0.6$. The same trend emerges in both the local pulse cases and global spectral cases. Therefore we conclude that the emergence of jet structure in a shallow water system is largely controlled by the energy injection rate and dissipation timescale. Given the values in Sections 2, colder giant planets like Jupiter might be located in the upperleft corner of Fig. 2 and brown dwarfs are probably in the lower-right corner.

Why jets emerge in turbulent, rotating stratified atmospheres remains incompletely understood. In an intrinsically $2 \mathrm{D}$ system approximating the stratified layer, when forcing occurs at small scales, it is generally expected that energy transfers to large scales via an "inverse energy cascade" (Vallis 2006). In the presence of damping, large coherent structures such as long-lived vortices and strong jets can develop only if energy can be transferred to large scales before it is dissipated. Two associated scales might be important. The first one is the "halting scale", associated with the peak wavenumber $\left(k_{h}\right)$ of the equilibrated kinetic energy (or available potential energy) spectrum. The second one is the "Rhines scale", $k_{\beta} \sim\left(\beta / U_{\mathrm{rms}}\right)^{1 / 2}$ (Rhines 1975), a characteristic scale
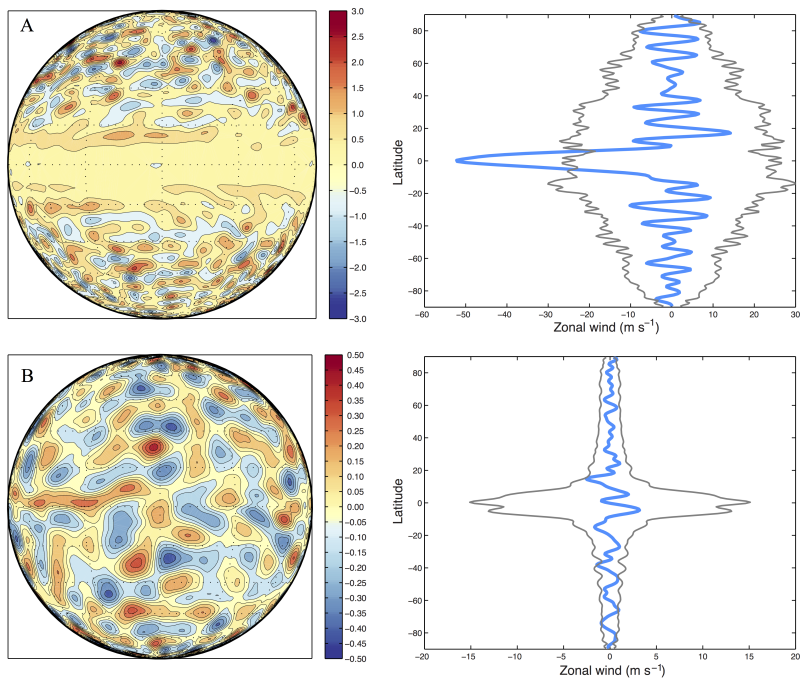

FIG. 1. - Two atmospheric regimes of brown dwarfs $(\Omega \sim$ $10^{-3} \mathrm{~s}^{-1}$ ) under forcing wavenumber 40 . Upper: jet case (case A); lower: isotropic turbulence case (case B). Left: geopotential anomaly map (in units of $10^{5} \mathrm{~m}^{2} \mathrm{~s}^{-2}$ ); right: zonal-mean zonal wind (blue) and standard deviation in longitude of the zonal wind at each latitude (gray). Both models have a small deformation radius of $\sim 10^{3} \mathrm{~km}$. 


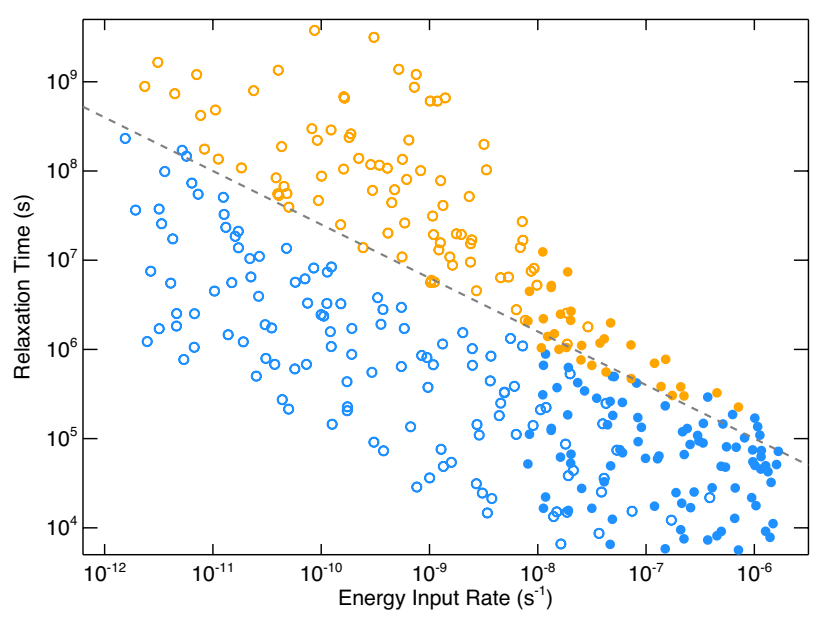

FIG. 2.- Regime diagram showing models exhibiting zonal jets (orange) and those without jets (blue) as function of energy input rate and relaxation timescale. Open circles: cases with local pulse forcing; filled circles: cases with global spectral forcing.
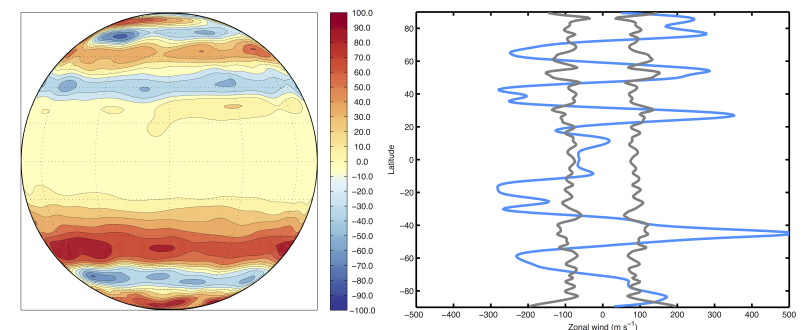

FIG. 3. - A brown dwarf $\left(\Omega \sim 10^{-3} \mathrm{~s}\right)$ simulation with $n_{f}=40$, $g h_{e q}=5 \times 10^{8} \mathrm{~m}^{2} \mathrm{~s}^{-2}, \tau_{\mathrm{rad}} \sim 10^{7} \mathrm{~s}, \tau_{\mathrm{s}} \sim 10^{5} \mathrm{~s}$, and $s_{m}=$ $10^{2} \mathrm{~m}^{2} \mathrm{~s}^{-3}$. Left: geopotential anomaly map (in units of $10^{5} \mathrm{~m}^{2}$ $\mathrm{s}^{-2}$ ); right: zonal-mean zonal wind (blue) and standard deviation in longitude of the zonal wind at each latitude (gray). This model has a large deformation radius of 0.3 planetary radii.

for significant east-west banding to occur, where $\beta$ is the latitudinal gradient of the Coriolis parameter and $U_{\mathrm{rms}}$ is the root mean square of the zonal wind velocity. Previous studies (e.g., Marcus et al. 2000; Danilov \& Gurarie 2001; Smith et al. 2002), most of which are non-divergent and hence significantly more idealized than the shallowwater system, have generally found that the necessary condition for zonal jets to emerge is $k_{h}<k_{\beta}$. Qualitatively, a similar criterion seems likely in the shallowwater system.

Interestingly, even the cases with equatorial jets are largely dominated by long-lived vortices at mid-to-high latitudes (Fig. 1). This likely results from the small deformation radius in these simulations, which is known to suppress the Rhines effect and jet formation under appropriate conditions (Okuno and Masuda 2003, Showman 2007). To test this hypothesis, we performed several simulation with thick layers and deformation radii up to half a planetary radius, which, when damping is sufficiently weak, indeed exhibit the formation of multiple jets both at the equator and at high latitude (Fig. 3). As 3D atmospheres exhibit multiple modes, including a baroclinic mode of small deformation radius and a barotropic mode of large deformation radius, it is possible that the atmospheric circulation on brown dwarfs with sufficiently long radiative time constants will qualitatively resemble a superposition of Fig. 3 and the top panel of Fig. 1.

\section{IMPLICATIONS FOR LIGHT CURVES}

We use the global shallow-water model to diagnose the long-term system behavior of brown dwarf atmospheres. Both the short-term and long-term behavior of our simulations, from hours to years, can help illuminate current and upcoming infrared light curve observations over various timescales (e.g., Artigau et al. 2009; Apai et al. 2013). As a proxy of the emergent flux, the height field $\bar{h}(t)$ averaged over the face-on hemisphere as a function of time $t$ can be obtained via:

$$
\bar{h}(t)=\int_{-\pi / 2+\Omega t}^{\pi / 2+\Omega t} \int_{-\pi / 2}^{\pi / 2} h(\phi, \lambda) R^{2} \cos ^{2} \phi \cos \lambda d \phi d \lambda
$$

In Fig. 4 we illustrate the time variations of geopotential anomalies of cases A and B (Fig. 1) after the systems reach statistically steady states. The geopotential anomalies are normalized to the equilibrium geopotential.

On multi-hour timescales, the simulated light curves are dominated by the rotation period, as atmospheric features rotate into or out of the Earth-facing hemisphere. The amplitude and shape of light curves remain similar over several rotation periods, but change dramatically over longer timescales (tens to hundreds of rotation periods). Frequently, the light curves exhibit double-peak or triple-peak features, qualitatively explaining the current observed light curve shapes (e.g., Artigau et al. 2009). The amplitude of the case without jet (case B) is less than the jet case (case A). The latter reaches up to several percent, similar to the observed flux variations (e.g., Artigau et al. 2009). The peak-to-peak amplitude of the fluctuations evolves on timescales of tens (case B) to hundreds (case A) of hours, shown by the blue envelope. In Fig. 1, the fractional variations in local height are order-unity, which is similar to the fractional variations in effective temperature between cloud and less-cloudy regions inferred from observations (Artigau et al. 2009, Radigan et al. 2012). Thus, if the patchy cloud structure on a brown dwarf had the same geometry as in our simulations, it should also produce lightcurve variations of $\sim 1 \%$ in spectral windows such as $J$.

A spectral analysis for both cases via Fast Fourier Transform (FFT) technique showed that the dominant mode is the rotational mode with a period of $\sim 1.6$ hours (Fig. 4, bottom row), and the other modes are the (even) multiples of the rotational mode as a fundamental frequency. This analysis suggests that, although the dynamics itself exhibits a range of inherent frequencies, the rotational modulation dominates the lightcurves.

\section{CONCLUSION}

We demonstrated using a global shallow-water model that zonal (east-west) jet streams and local vortices can form under conditions appropriate to brown dwarfs. The existence and properties of the jets crucially depend on the radiative damping timescale and the rate at which convection injects energy into the atmosphere. Strong internal heat flux (i.e., strong convective forcing) and weak radiative dissipation favors the formation of largescale jets in the atmosphere, as shown in our simulations, whereas a weaker forcing and stronger dissipation will halt the formation of zonal jets and favor the generation of (horizontally) isotropic turbulence. 

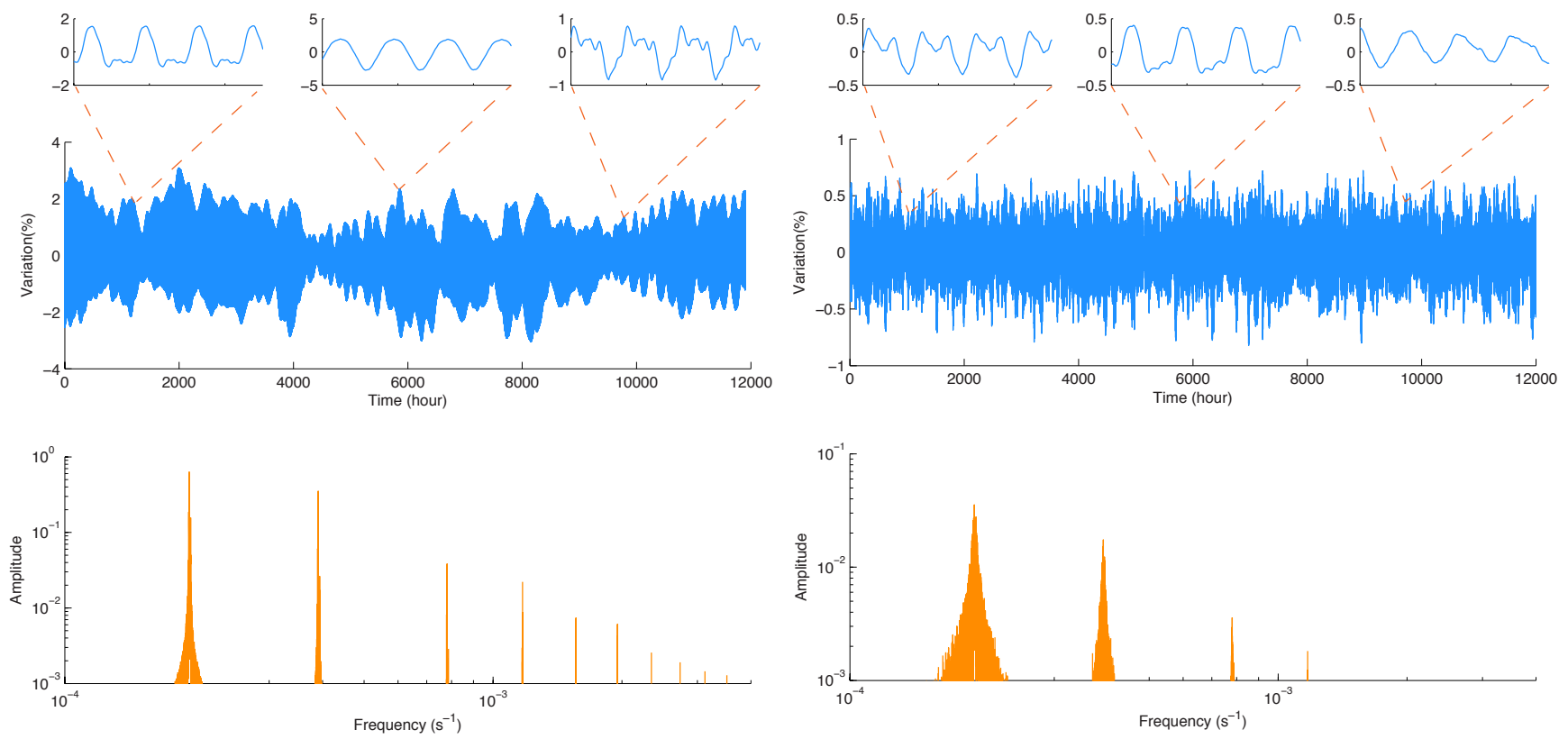

Fig. 4.- Light curve analysis for case A (left) and case B (right). Upper: time variability of face-on geopotential anomaly, with three zoom-in charts; Lower: Single-sided amplitude spectra via FFT. Only noise exists at frequencies shorter than $10^{-4} \mathrm{~s}^{-1}$.

The radiative timescale for most brown dwarfs above $1000 \mathrm{~K}$ is $\sim 10^{5} \mathrm{~s}$ or less, and longer with colder atmospheres. The rate of energy injection at the RCB is less certain, which depends on the forcing mechanisms, either from the dry and moist convective processes, or from the upward propagating atmospheric waves. A crude estimate of a typical brown dwarf implies that its energy injection rate is several orders of magnitude larger than that on Jupiter.

Our simulations suggest that many hot brown dwarfs may be eddy/turbulence dominated rather than jet dominated at typical IR photosphere levels. If a brown dwarf is cold enough, such as Y dwarfs, or the damping timescale is long, or the forcing is strong enough, zonal jets should form, as seen on Jupiter. Nevertheless, it is difficult to precisely relate our forcing and damping parameters to the brown dwarf spectral sequence, as $\epsilon$ and $\tau_{\text {rad }}$ will depend on the detailed nature of the convection, the thickness of the layer communicating with the photosphere (which may include the top portion of the convection zone), and other factors. While our finding of a regime transition is robust and should carry over to the full 3D system, these uncertainties imply that more realistic three-dimensional models will be necessary to pin down precisely where the transition falls in the brown dwarf spectral sequence. Despite these uncertainties, it is clear that Jupiter is in the jet-dominated regime, providing some confirmation of our findings.

The long-time integration of the shallow water system provides a new tool to understand the observed light curve variations. We found that our simulated brown dwarf atmospheres are dominated by the rotational modulation in short-term light curves, with lightcurve shapes that vary from single to multi-peaked periodic structures and amplitudes of a few percent, qualitatively consistent with recent observed infrared flux variations.

\section{ACKNOWLEDGEMENTS}

This research was supported by Bisgrove Scholar Program to X. Z. and NSF grant AST1313444 to A.P.S. The authors wish to thank the International Summer Institute for Modeling in Astrophysics (ISIMA) where part of the model was developed.

\section{REFERENCES}

Apai, D., Radigan, J., Buenzli, E., et al. 2013, ApJ, 768, 121 Artigau, É., Bouchard, S., Doyon, R., \& Lafrenière, D. 2009, ApJ, 701, 1534

Burrows, A., Sudarsky, D., \& Hubeny, I. 2006, ApJ, 640, 1063

Cho, J. Y.-K., \& Polvani, L. M. 1996, Physics of Fluids, 8, 1531

Cooper, C. S., Sudarsky, D., Milsom, J. A., Lunine, J. I., \& Burrows, A. 2003, ApJ, 586, 1320

Crossfield, I., Biller, B., Schlieder, J., et al. 2014, Nature, 505, 654

Danilov, S., \& Gurarie, D. 2001, Phys. Rev. E, 63, 020203

Freytag, B., Allard, F., Ludwig, H.-G., Homeier, D., \& Steffen, M. 2010, A\&A, 513, A19

Hack, J. J., \& Jakob, R. 1992, Description of a global shallow water model based on the spectral transform method (National Center for Atmospheric Research)

Helling, C., Oevermann, M., Lüttke, M. J. H., Klein, R., \& Sedlmayr, E. 2001, A\&A, 376, 194
Kiladis, G. N., Wheeler, M. C., Haertel, P. T., Straub, K. H., \& Roundy, P. E. 2009, Reviews of Geophysics, 47

Knapp, G. R., Leggett, S. K., Fan, X., et al. 2004, AJ, 127, 3553 Leggett, S. K., Saumon, D., Marley, M. S., et al. 2007, ApJ, 655, 1079

Mapes, B., Tulich, S., Lin, J., \& Zuidema, P. 2006, Dynamics of atmospheres and oceans, 42, 3

Marcus, P. S., Kundu, T., \& Lee, C. 2000, Physics of Plasmas, 7, 1630

Morley, C. V., Fortney, J. J., Marley, M. S., et al. 2012, The Astrophysical Journal, 756, 172

Okuno, A., \& Masuda, A. 2003, Physics of Fluids, 15, 56

Radigan, J., Jayawardhana, R., Lafrenière, D., et al. 2012, The Astrophysical Journal, 750, 105

Reiners, A., \& Basri, G. 2008, The Astrophysical Journal, 684, 1390 
Rhines, P. B. 1975, Journal of Fluid Mechanics, 69, 417

Sadourny, R. 1975, Journal of Atmospheric Sciences, 32, 680

Saumon, D., Marley, M. S., Cushing, M. C., et al. 2006, ApJ, 647,552

Showman, A. P. 2007, Journal of Atmospheric Sciences, 64, 3132

Showman, A. P., \& Kaspi, Y. 2013, ApJ, 776, 85

Showman, A. P., \& Polvani, L. M. 2011, ApJ, 738, 71
Smith, K. S., Boccaletti, G., Henning, C. C., et al. 2002, Journal of Fluid Mechanics, 469, 13

Tsuji, T. 2002, ApJ, 575, 264

Vallis, G. K. 2006, Atmospheric and Oceanic Fluid Dynamics, doi: $10.2277 / 0521849691$

Vasavada, A. R., \& Showman, A. P. 2005, Reports on Progress in Physics, 68, 1935 